\title{
Diazepam-binding inhibitor/acyl-CoA-binding protein mRNA and peripheral benzodiazepine receptor mRNA in endocrine and immune tissues after prenatal diazepam exposure of male and female rats
}

\author{
B Bürgi, W Lichtensteiger and $M$ Schlumpf \\ Institute of Pharmacology and Toxicology, University of Zürich, CH-8057 Zürich, Switzerland \\ (Requests for offprints should be addressed to M Schlumpf, Institute of Pharmacology and Toxicology, University of Zürich, Winterthurerstrasse 190, \\ CH-8057 Zürich, Switzerland; Email: schlumpm@pharma.unizh.ch)
}

\begin{abstract}
Peripheral benzodiazepine (BDZ) receptor (PBR) and diazepam-binding inhibitor/acyl-CoA-binding protein (DBI/ACBP) characterized as a ligand at central BDZ receptors, at $\mathrm{PBR}$ with involvement in the regulation of steroidogenesis, and as an intracellular acyl-CoA transporter, are both known to interact with BDZ in adult systems. We investigated their expression after prenatal exposure to BDZ. Diazepam $(1 \cdot 25 \mathrm{mg} / \mathrm{kg}$ per day s.c.) was administered to time-pregnant Long Evans rats from gestational day (GD) 14 to 20. Expression of mRNAs encoding for PBR and for DBI/ACBP was studied in the same animals with ${ }^{33} \mathrm{P}$-labeled 60 mer oligonucleotides (oligos) by in situ hybridization at GD20, and with ${ }^{32} \mathrm{P}$-labeled oligos by Northern blot in steroidogenic and immune organs at postnatal day (PN) 14 and in adult offspring. Prenatal diazepam increased DBI/ACBP mRNA expression in male fetal adrenal and in fetal and
\end{abstract}

PN14 testis. Thymus exhibited increased DBI/ACBP mRNA in male fetuses and in adult female offspring, and reduced organ weight at PN14 in both sexes. In female spleen, an increase in DBI/ACBP mRNA and a decrease in PBR mRNA was seen at PN14. Apart from the finding in spleen, no drug-induced changes in PBR mRNA were observed. The effects of prenatal diazepam were superimposed on treatment-independent sex differences in DBI/ACBP mRNA and PBR mRNA expression. Our data indicate that expression of DBI/ACBP mRNA in steroidogenic and immune organs can be affected by exposure to BDZ during ontogeny, while PBR mRNA expression appears to be less sensitive. They further reveal marked sex differences in the developmental patterns of the two proteins during pre- and postpubertal ontogeny.

Journal of Endocrinology (2000) 166, 163-171

\section{Introduction}

The development of the central nervous system, neuroendocrine system and immune system can be modulated by prenatal exposure to benzodiazepines (BDZ) (Kellogg 1988, Schlumpf et al. 1995). While actions of BDZ on the fetal central nervous system may be mediated by centraltype BDZ-binding sites on $\gamma$-aminobutyric acid type A receptors $\left(\mathrm{GABA}_{\mathrm{A}}\right.$ receptors) (Schlumpf et al. 1983, 1995), the peripheral BDZ receptor (PBR), which is expressed by a majority of fetal tissues (Bürgi et al. 1999), might represent a site of action of BDZ on developing non-neural structures such as immune and endocrine systems. The PBR, which binds BDZ and isoquinoline carboxamide derivatives, has been localized to the outer mitochondrial membrane and cell membrane (Woods et al. 1996, Papadopoulos et al. 1997). In steroidogenic tissues, it was found to function as a carrier for cholesterol from the outer to the inner lipid monolayer of the outer mitochondrial membrane (Garnier et al. 1994), the ratelimiting step of steroid hormone biosynthesis (Jefcoate et al. 1992).

Evidence for a possible involvement of PBR in developmental BDZ toxicity stems from investigations on the immune system. Delayed deficiencies in immune functions of rat offspring after prenatal diazepam exposure include reduced lymphocyte proliferation and antibody formation, altered cytokine patterns and impaired host defense (reviewed by Schlumpf et al. 1995). A reduction of mitogen-induced lymphocyte proliferation was also found after prenatal treatment with the specific PBR agonist Ro5-4864 (Schlumpf et al. 1990). Maximal binding capacity of the PBR ligand $\left[{ }^{3} \mathrm{H}\right] \mathrm{PK} 11195$ was markedly reduced in macrophage membranes of adult prenatally diazepam-exposed rats (Schlumpf et al. 1993b). 
Developmental toxicity might also result from changes in signaling molecules interacting with target sites of BDZ. One such factor is the diazepam-binding inhibitor/ acyl-CoA-binding protein (DBI/ACBP), which is strongly expressed in embryonic and fetal rat tissues (Bürgi et al. 1999). This $10 \mathrm{kDa}$ polypeptide is a ligand at central-type BDZ-binding sites on $\mathrm{GABA}_{\mathrm{A}}$ receptors (Guidotti et al. 1983) as well as at PBR (Papadopoulos et al. 1997), but it also binds acyl-CoA (Mogensen et al. 1987, Mandrup et al. 1992), functions as an intracellular acylCoA transporter (Knudsen et al. 1993), and regulates acyl-CoA:cholesterol acyltransferase (Kerkoff et al. 1997). The peptide was found to be involved in the control of fatty acid, glycerolipid (Rasmussen et al. 1993, 1994) and phospholipid synthesis (Fyrst et al. 1995). Transcription of the DBI/ACBP gene is enhanced by sterol regulatory element (SRE)-binding proteins (SREBPs) (Swinnen et al. 1998). Interactions between BDZ and DBI/ACBP occur not only at the two BDZ receptor sites, but also in lipid metabolism. Diazepam has been found to interfere with steroidogenesis in liver (Bell 1985), and to compete with oleolyl-CoA for a common binding site on microsomal membranes (Kerkhoff et al. 1997).

In the adult, DBI/ACBP and PBR are both upregulated by chronic treatment with diazepam, PBR also by selective PBR agonists (Mocchetti \& Santi 1991, Weizman et al. 1997). Given the widespread presence of mRNAs encoding for DBI/ACBP and PBR in embryonic and fetal rat tissues (Bürgi et al. 1999) and the changes in PBR-binding sites on macrophages after prenatal diazepam exposure (Schlumpf et al. 1993b), we hypothesized that the regulation of one or both of these proteins might be influenced by prenatal BDZ exposure. Our study revealed tissue- and stage-specific alterations mainly in DBI/ACBP mRNA expression, with little change in PBR mRNA, and marked postnatal sex differences.

\section{Materials and Methods}

\section{Animals and treatment schedule}

The study was conducted on time-pregnant Long Evans rats from our colony (originating from Møllegard Breeding \& Research Center, Denmark), mated between 1600 and $1900 \mathrm{~h}$, and kept in groups of two to four under controlled conditions (lights on $0200-1600 \mathrm{~h}, 22^{\circ} \mathrm{C}$, and standard diet (NAFAG 850, Olten, Switzerland) and water freely available) following confirmation of sperm-positive vaginal smears. The stage $24 \mathrm{~h}$ after the mating period was defined as gestational day (GD) 1 , the day of birth as postnatal day (PN) 1 (=GD23). Diazepam, $1 \cdot 25 \mathrm{mg} / \mathrm{kg}$ (Hoffmann La Roche, Basel, Switzerland) dissolved in sterilized olive oil, or olive oil was injected s.c. once daily from GD14 to GD20 (seven injections). DBI/ACBP mRNA and PBR mRNA expression was studied in the same animals. At GD20, dams were anesthetized with chloral hydrate
(400 mg/kg s.c.) followed by ether, $4-6 \mathrm{~h}$ after the last diazepam injection. Fetuses were frozen in isopentane cooled by liquid nitrogen and stored in liquid nitrogen. Offspring were decapitated at PN14 or in adulthood (age 2 months). Adrenal gland, testis, thymus and spleen were weighed (wet weight), and frozen and stored in liquid nitrogen.

\section{In situ hybridization}

In situ hybridization was performed on $10 \mu \mathrm{m}$ serial sagittal sections through GD20 male and female fetuses as previously described (Bürgi et al. 1999). In order to increase the signal, two specific 60 mer oligonucleotides (oligos) for the PBR (PBR oligo I: 5'-GTG GCG GTT GCC ACC CCA CTG ACA AGC ATG AGG TCC ACC AAA GCC CAG CCC ATC AAA AAA-3'; PBR oligo II: 5'CTG CAA GCT AGC ATA CCA GCG GAG GCC CTC ACC ACG CAC AAA GTA GGC TCC CAT GAA GCC-3') and two oligos for the DBI/ACBP (DBI/ ACBP oligo I: 5'-GCC CTT GAG GCC CAA CAG CCC CGG CCCG ATC TGT GTT TAG ATC GCC CAC AGT AGC AAA AAA-3'; DBI/ACBP oligo II: 5'-CAT ACT GGC GAG GTG ACC TGG ATG CTG AAA GCG CGG GAG CAA GCT CAG AAG AGG CTT CGT-3') were selected, and synthesized by Microsynth, CH-9436 Balgach, Switzerland. PBR oligo I and $\mathrm{DBI} / \mathrm{ACBP}$ oligo II have been used in the previous study (Bürgi et al. 1999). Sequences were chosen from a gene bank data according to GC-content (PBR oligo I $55 \%$, PBR oligo II 60\%, DBI/ACBP oligo I 56\%, DBI/ACBP oligo II 58\%). The PBR oligos hybridize specifically to the $18 \mathrm{kDa}$ isoquinoline-binding protein of the PBR. DBI/ACBP oligos I and II were also compared with the amino acid sequence of membrane-associated DBI (MA-DBI) using a GC (Genetics Computer Group, University of Wisconsin sequence analysing package, Madison, WI, USA) program (Best fit, Compare, DotPlot). For oligo I, the analysis revealed a sequence of 24 bases (29-52) with 17 matches and 7 mismatches to the sequence 50-73 of the 252-base MA-DBI (Bürgi et al. 1999); for oligo II no apparent homologies to MA-DBI were observed. With the stringent hybridization conditions used, the probability of hybridization of the labeled oligo to MA-DBI mRNA is expected to be very low. The oligos, PAGE purified by Microsynth, were labeled at their $3^{\prime}$-end with $\left[\alpha-{ }^{33} \mathrm{P}\right] \mathrm{dATP}$ (DuPont-New England Nuclear Corp., Boston, MA, USA) using terminal transferase (Boehringer-Mannheim, CH-6343 Rotkreuz, Switzerland). The labeled oligo probe was controlled by PAGE (Bürgi et al. 1999). The two oligos for either DBI/ACBP or PBR mRNA were pooled in the hybridization solution (350 000 c.p.m. oligo I +350 000 c.p.m. oligo II in $80 \mu \mathrm{l})$.

Tissue sections were incubated overnight at $43{ }^{\circ} \mathrm{C}$ with hybridization solution $(80 \mu \mathrm{l} /$ section) containing the 
two oligos, washed and apposed to Hyperfilm $\beta$ max (Amersham, CH-8600 Dübendorf, Switzerland) (Bürgi et al. 1999). Sections with total hybridization signals and non-specific signals from treated and control animals of both sexes were arranged in one Kodak cassette containing 72 sections (Kodak, Zürich, Switzerland). Films were exposed for 3 months at $4{ }^{\circ} \mathrm{C}$ and developed manually using solutions from Kodak. All sections were counterstained with 1\% toluidine blue in PBS following film exposure. Specificity was assessed by adding a 100-fold excess of each of the two unlabeled oligos to the incubation mixture. PBR and DBI/ACBP mRNA localization was analyzed on neighboring sections of the same animal according to the following sequence: DBI/ACBP 'hot', DBI/ACBP 'cold', PBR 'hot', PBR 'cold'.

\section{Image analysis}

Relative optical densities (ROD) were measured in an MCID image analysis system (Imaging Research, St Catharines, Ontario, Canada). Each measurement was carried out in duplicate. Specific ROD of different tissues were obtained by subtracting background ROD of the same tissue measured in a neighboring section, incubated with a 100-fold excess of the two cold oligos, from the total ROD of the tissue. Mean ROD values were calculated for each tissue of each fetus. From these, mean tissue values of control and diazepam groups were obtained.

\section{Northern blots}

Thymus, spleen, adrenals and testes were dissected at PN14 and at 2 months. Adult females were analyzed in estrus confirmed by vaginal smears. All organs were stored in liquid nitrogen. RNA was extracted from tissues using the Rneasy Mini/Midi Total RNA kit of Qiagen, Basel, Switzerland, according to the protocol provided by the manufacturer, and stored at $-80{ }^{\circ} \mathrm{C}$. Precipitated RNA was further processed according to standard procedures (Sambrook et al. 1989). Hybridization of RNA transferred to POSITIVE membrane (Appligene, Oncor, Switzerland) was carried out overnight at $42{ }^{\circ} \mathrm{C}$ with either $\left[\alpha-{ }^{32} \mathrm{P}\right]$ dATP-labeled DBI/ACBP oligo I $\left(50 \times 10^{6}\right.$ c.p.m. $/ 30 \mathrm{ml})$ or $\left[\alpha^{32} \mathrm{P}\right] \mathrm{dATP}-$ labeled PBR oligo I $\left(50 \times 10^{6}\right.$ c.p.m. $\left./ 30 \mathrm{ml}\right)$. Membranes were washed, apposed to Hyperfilm MP overnight at $-80{ }^{\circ} \mathrm{C}$ and developed manually the next day.

DBI/ACBP- and PBR-hybridized membranes were rehybridized with an 80 mer actin oligo (smooth muscle actin; 5'-CTCGTTGTAG AAGGAGTGGT GCCAG ATCTT CTCCATGTCA TCCCAGTTCG TGATG ATGCC GTGCTCAATG GGGATAAAAT-3') to calibrate the DBI/ACBP and PBR bands. Membranes were apposed to films and developed as mentioned above. The resulting DBI/ACBP, PBR and actin bands were converted into optical density units using a densitometer calibrated by an internal calibration program (Computing Densitometer, Modul 300A, Molecular Dynamics, Groningen, The Netherlands). Optical density readings for DBI/ACBP and PBR bands were corrected by the corresponding optical density value of the actin band. PBR mRNA was below the detection limit in postnatal testis.

\section{Data analysis}

Multivariate ANOVA of ROD values from in situ hybridization, of corrected optical density readings from Northern blots, and of organ wet weights of individual animals was carried out separately for different developmental stages, followed by the Bonferroni test for pairwise comparisons.

\section{Results}

\section{$D B I / A C B P$ mRNA}

DBI/ACBP mRNA expression exhibited tissue-, stageand sex-specific patterns. At the end of the prenatal treatment period, GD20, the signal was increased by $35-86 \%$ in thymus and adrenal of diazepam-treated male fetuses and in their testes (Table 1, Figs 1 and 3), whereas no treatment effect was seen in females. Spleen could not be measured at that stage. At PN14, DBI/ACBP mRNA was even more elevated in the testes of prenatally diazepam-exposed offspring, and was also increased in the spleen of treated females (Figs 2 and 3). In adulthood, DBI/ACBP mRNA was significantly increased in the thymus of female, prenatally diazepam-exposed offspring, with a similar tendency in the spleen, while no persistent changes were noted in males.

These drug-induced changes were superimposed on sex differences in DBI/ACBP mRNA expression (Table 1, Fig. 3). Irrespective of prenatal treatment, mRNA levels in thymus of males were significantly higher than those of females at PN14 (Fig. 2) and lower in adulthood. Adult males further exhibited higher DBI/ACBP mRNA levels in spleen and adrenal. In addition, two sex differences were restricted to treated animals, i.e. higher adrenal levels in female fetuses and lower splenic levels in PN14 females. These two differences result from the sex-specific treatment effects.

\section{PBR $m R N A$}

The only significant effect of prenatal diazepam exposure was a decrease in PBR mRNA in the spleen of female offspring at PN14, i.e. at the stage when DBI/ACBP mRNA was selectively increased in the spleen of treated females (Table 2). PBR mRNA expression also exhibited treatment-independent sex differences (Table 2). At PN14, males exhibited $710-845 \%$ of female levels in 
Table 1 Effect of prenatal diazepam exposure on DBI/ACBP mRNA expression (mean \pm S.E.M. $)^{1}$

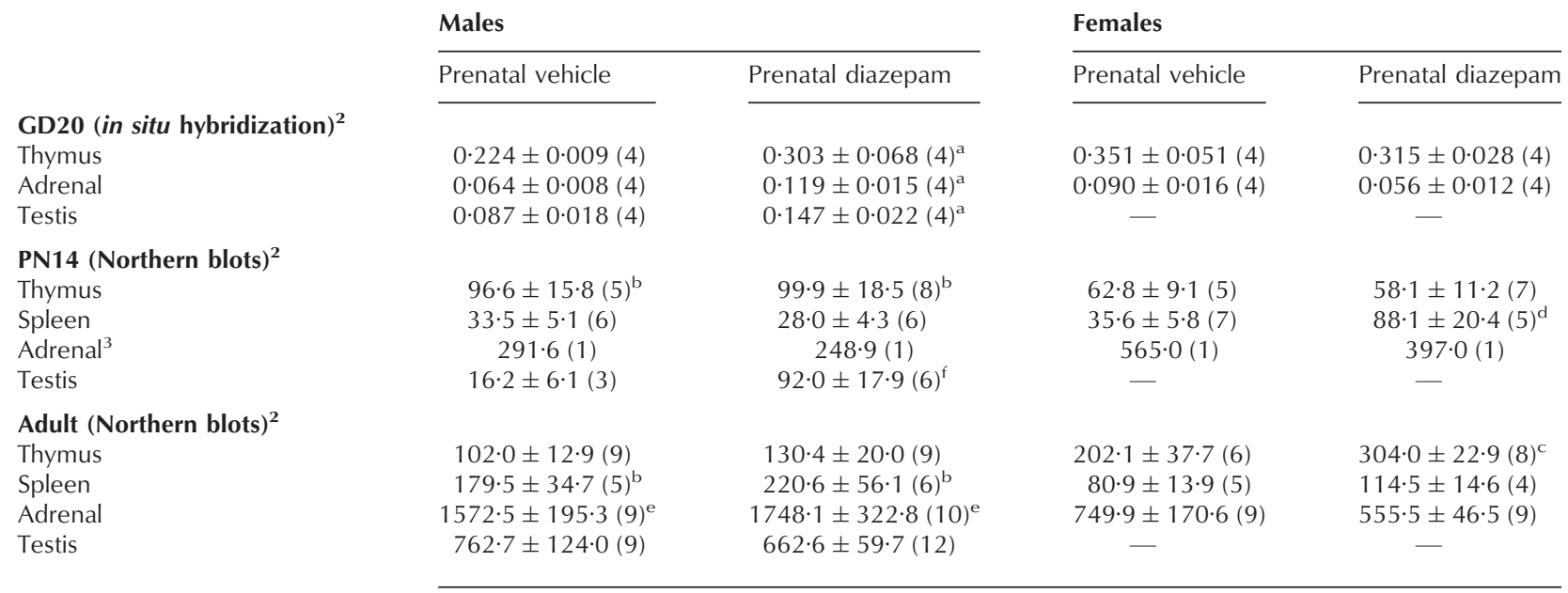

${ }^{1} 4-12$ litters.

${ }^{2}$ In situ hybridization: (ROD); Northern blots: corrected optical density.

${ }^{3}$ Pooled values from $10-15$ animals.

${ }^{a}$ Group of thymus, adrenal and testis in males different from control $P=0 \cdot 020$.

${ }^{b}$ Sex difference for control and treated groups $P=0 \cdot 025$.

${ }^{\mathrm{C}}$ Different from control $P=0 \cdot 036$.

${ }^{\mathrm{d}}$ Female diazepam different from control $P=0 \cdot 007$, sex difference in male and female diazepam groups $P=0 \cdot 004$.

eSex difference ANOVA $P=0 \cdot 0001$, Bonferroni controls $P=0 \cdot 075$, diazepam $P=0 \cdot 002$.

fDifferent from control $P=0 \cdot 024$.

thymus and $18-23 \%$ of females levels in spleen. Adult adrenal values were slightly but significantly lower in males.

\section{Organ weights}

The only treatment effect was noted at PN14 with a $10 \%$ decrease of thymus weight in prenatally exposed offspring of both sexes (males: prenatal vehicle 0.09 \pm 0.004 g S.E.M. $(n=16)$, prenatal diazepam $0 \cdot 08 \pm 0.004 \mathrm{~g}(n=18)$, females: prenatal vehicle $0 \cdot 09 \pm 0 \cdot 005 \mathrm{~g}(n=15)$, prenatal diazepam $0 \cdot 08 \pm 0 \cdot 004 \mathrm{~g}(n=19), P=0 \cdot 012)$. No changes were seen in the remaining organ weights and in body weight (data not shown).

\section{Discussion}

The present investigation revealed distinct tissue-, stageand sex-specific changes in DBI/ACBP mRNA expression after prenatal diazepam exposure, with little effect on PBR mRNA. The two PBR oligos used here selectively detect mRNA encoding for the isoquinoline-binding protein. The two oligos designed for detection of DBI/ ACBP mRNA were also compared with the amino acid sequence of a related protein, MA-DBI (Todaro et al. 1991) using a GCG program. Because the probability of hybridization of the two oligos to MA-DBI mRNA was very low (Bürgi et al. 1999, and this study), we assume that the hybridization data essentially reflect the presence of
DBI/ACBP mRNA. Since both, DBI/ACBP and PBR mRNA were studied in the same animals, relative changes in expression can be compared.

$\mathrm{DBI} / \mathrm{ACBP}$ mRNA expression increased markedly in testis as well as in adrenal and spleen of males from the immature (P14) to the adult stage, resulting in significantly higher levels in adult males as compared with females, irrespective of prenatal treatment. This difference is probably due to androgens. Swinnen et al. (1994, 1996) observed an enhancement of DBI/ACBP mRNA expression by androgens in rat adrenal, male accessory sex organs, and lacrimal and salivary glands. However, there also appear to exist different regulatory patterns. In thymus, DBI/ACBP mRNA expression switched from higher levels in immature (PN14) males to lower levels in adult males (again independent of prenatal treatment). In liver and kidney, DBI/ACBP appears not to be responsive to androgens at all (Swinnen et al. 1994, 1996). The androgen effect on DBI/ACBP transcription may be indirect (Swinnen et al. 1994). Several regulatory elements have been located in the promoter region of the rat gene encoding for DBI/ACBP (Elholm et al. 1996). Data from $\mathrm{LNCaP}$ prostate cancer cells indicate that the DBI/ACBP promoter contains a functional SRE. Activation of SREBPs by androgen treatment was accompanied by increased DBI/ACBP mRNA expression in these cells (Swinnen et al. 1998).

The density of PBR-binding sites also can be modulated in some tissues by androgen or anti-androgen 

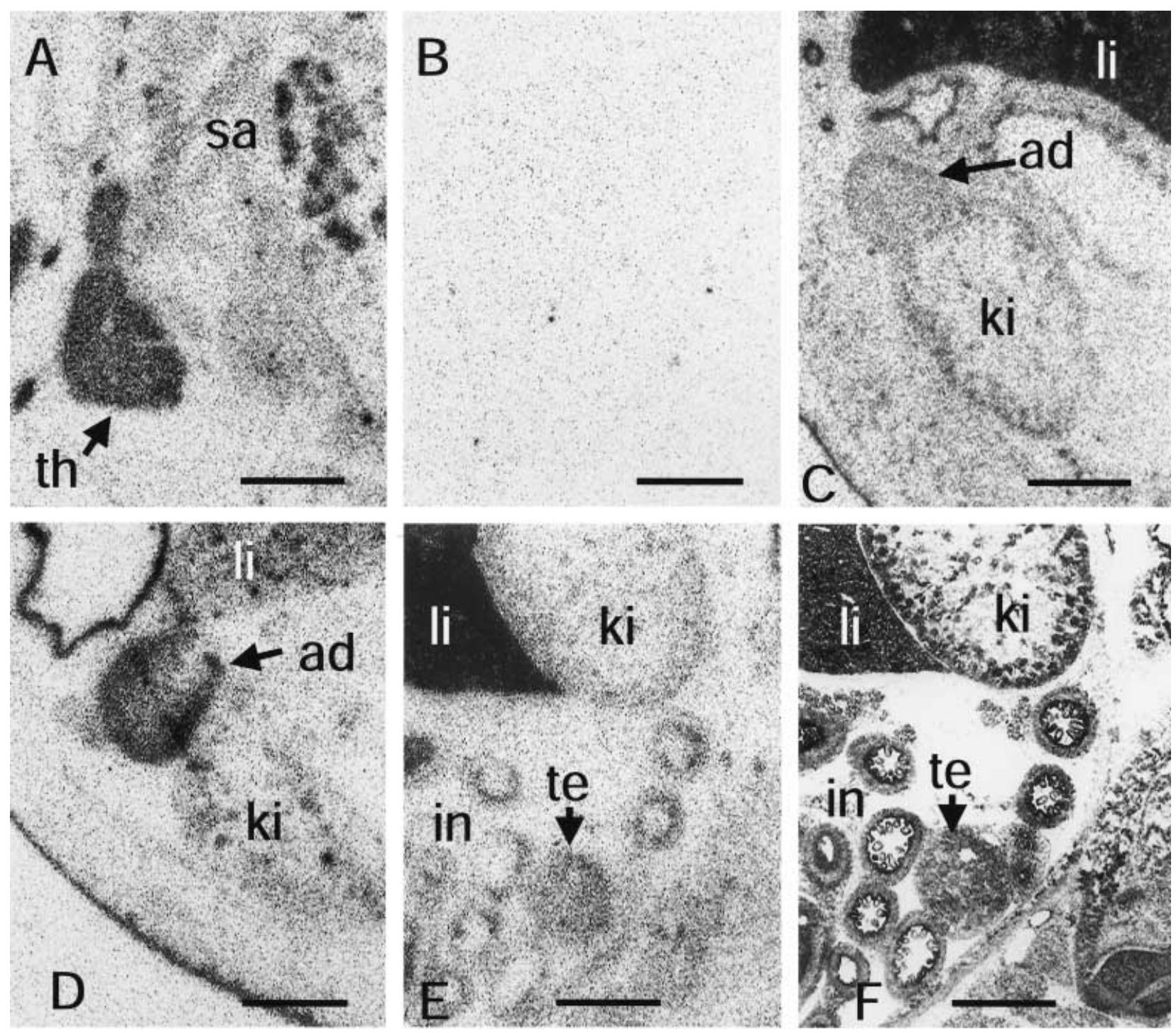

Figure 1 Expression of DBI/ACBP mRNA and PBR mRNA in untreated male rat fetus at GD20. In situ hybridization of sagittal sections. $\mathrm{DBI} / \mathrm{ACBP}$ mRNA expression in thymus (th) (A), adrenal (ad) (C), and testis (te) (E). Additional labeled structures: kidney (ki), liver (li), small intestine (in), submandibular salivary gland (sa). (B) Section adjacent to (A) incubated with labeled oligos in the presence of a 100-fold excess of unlabeled oligos. (F) Histology of the section shown in (E). Expression of PBR mRNA in adrenal (ad) is shown in (D). Bar=1 mm. ROD was measured across the entire surface of the various organs.

treatment (Gavish 1995), and has been found to increase in the adrenal after prolonged testosterone administration (Amiri et al. 1991). However, the steady-state PBR mRNA levels we determined in the adult adrenalreflecting levels in the absence of exogenous steroid treatment - were slightly but significantly lower in males than in females. An interesting but unexplained finding is the markedly higher PBR mRNA expression in immature (P14) male thymus which parallels the elevated DBI/ ACBP mRNA level in this organ.

Prenatal exposure to diazepam caused immediate and delayed changes in DBI/ACBP mRNA expression which were sex-dependent. At the end of the treatment period, GD20, diazepam-exposed male fetuses exhibited increased DBI/ACBP mRNA in thymus, adrenal and testis, whereas levels in female thymus and adrenal were unaffected. The drug-induced increase in mRNA expression resembles the situation in adult brain, where DBI/ACBP synthesis was upregulated following chronic diazepam (Mocchetti \& Santi 1991); however, its restriction to males suggests a dependence on fetal steroid hormones.

The increased expression of DBI/ACBP mRNA in testis continued beyond the treatment period to become 
Testis

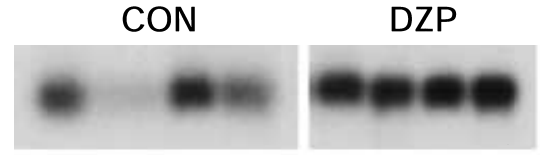

\section{Spleen}

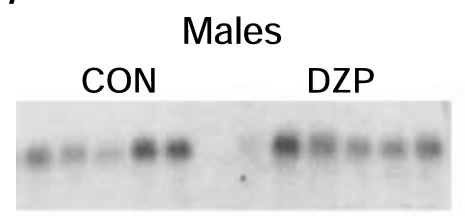

\section{Thymus}

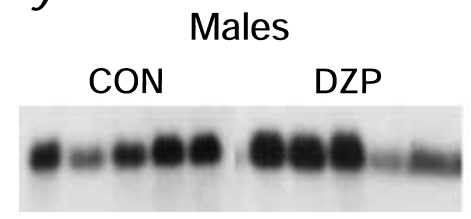

Females CON DZP

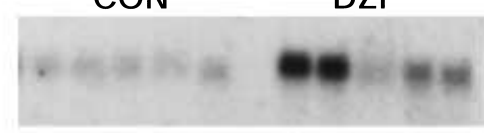

Females

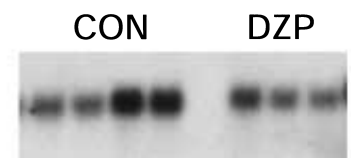

Figure 2 Representative Northern blots for DBI/ACBP mRNA of testis, spleen and thymus from male and female rat offspring at PN14. CON: prenatal vehicle; DZP: prenatal diazepam treatment $(1 \cdot 25 \mathrm{mg} / \mathrm{kg}$ per day s.c., GD14-20). Each blot represents the organ of one animal.

even stronger at P14, but had disappeared in adulthood. This pattern suggests a relationship with the switch from the fetal to the adult Leydig cell population; in the rat, the progenitors of adult Leydig cells arise during the third week of postnatal life (Benton et al. 1995). Thus, the prenatal diazepam treatment probably affected the fetal Leydig cell population but remained without effect on the adult population that originated long after the cessation of drug treatment. Adrenal DBI/ACBP mRNA expression had already normalized at PN14.

Spleen exhibited a marked increase in DBI/ACBP mRNA expression in prenatally diazepam-exposed female offspring at PN14; levels tended to remain slightly elevated also in adulthood, but the effect was not significant. The female PN14 spleen also exhibited a druginduced change (decrease) in PBR mRNA expression. We previously observed a reduction in $\mathrm{PBR}\left(\left[{ }^{3} \mathrm{H}\right] \mathrm{PK}\right.$ 11195-binding sites) on splenic macrophages of adult female offspring after the same prenatal treatment (Schlumpf et al. 1993b), but we failed to detect a change in PBR mRNA of adult spleen. It seems possible that the interindividual variability of Northern blot data prevented detection of subtle differences. Also, receptor binding was studied on a defined cell population, macrophages, whereas mRNA was analyzed in whole spleen. Indeed, $\mathrm{B}_{\max }$ of $\left[{ }^{3} \mathrm{H}\right] \mathrm{PK} 11195$ binding to membranes prepared from a mixture of splenic cells of adult rats, rather than from macrophages, was not changed following prenatal diazepam (Schlumpf et al. 1993b).
Data on proliferation and cytokine regulation of splenic cells point to dysfunctions of both splenic macrophages and lymphocytes and of the autonomic innervation of spleen (Schlumpf et al. 1995). Sex differences in delayed effects of prenatal diazepam exposure were found, in pre- and peripubertal rats, for the macrophage-derived cytokine interleukin-1 but not for the lymphocyte cytokine interleukin-2 (Schlumpf et al. 1993a). Moreover, antibody formation against sheep red blood cells, studied in prepubertal rats, was more strongly reduced after prenatal diazepam in females than in males, and the sympathetic control of spleen was altered only in female offspring (Bütikofer et al. 1993). These effects cannot be directly related to the present data, but they further support the role of sex in the delayed drug effects.

Thymus belongs to the organs with high levels of DBI/ACBP mRNA, PBR mRNA and PBR-binding sites during mid- and late fetal life, i.e. at a critical phase of differentiation which was included in the treatment period (Schlumpf et al. 1990, Bürgi et al. 1999). The complex drug-induced changes, with increased DBI/ACBP mRNA expression in male fetuses and in adult female offspring, and a reduction of organ weight at PN14 in both sexes, indicate that the development of this immune organ was disturbed by prenatal diazepam until adulthood.

These data indicate that exposure to a low dose of diazepam during a limited period of fetal life can affect the expression of DBI/ACBP mRNA in steroidogenic and immune organs during pre- and postnatal ontogeny, in a 


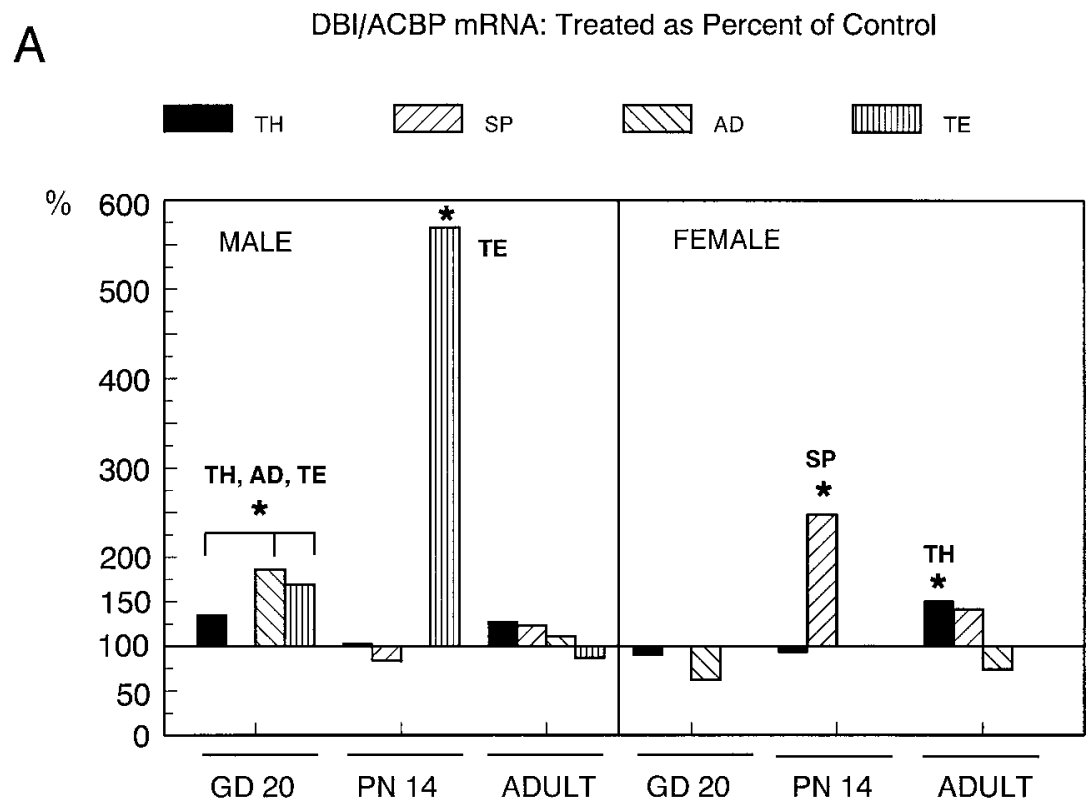

B

DBI/ACBP mRNA: Male as Percent of Female
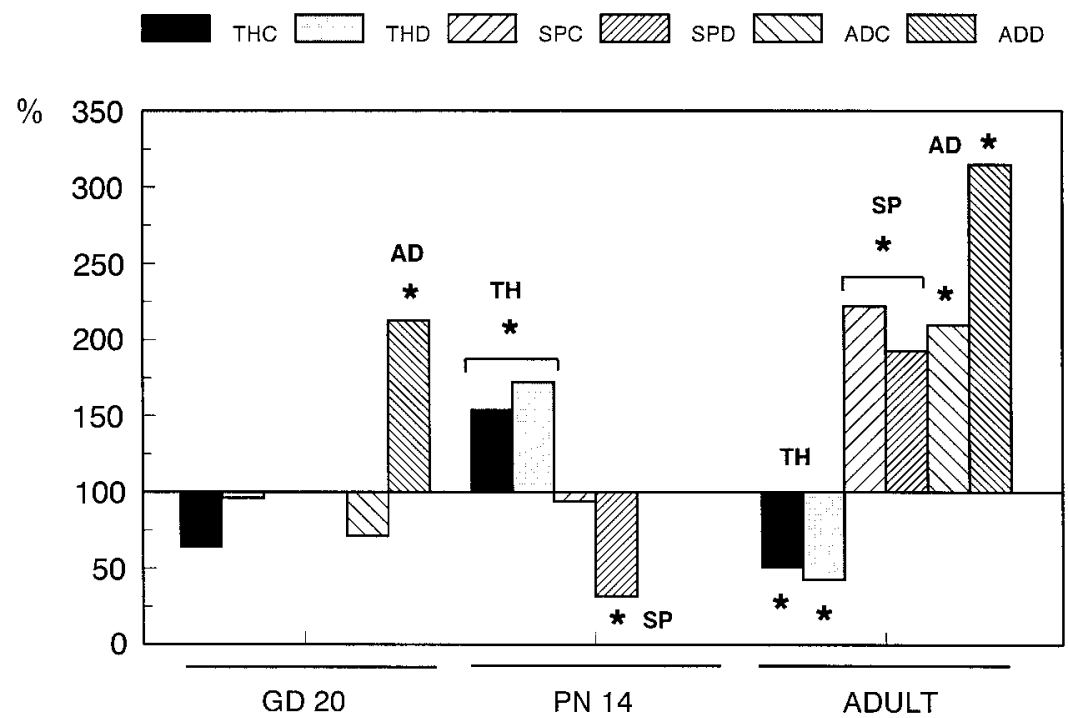

Figure 3 Expression patterns of DBI/ACBP mRNA in rats at GD20, PN14 and in adulthood (2 months). (A) Changes in DBI/ACBP mRNA induced by prenatal exposure to diazepam $(1.25 \mathrm{mg} / \mathrm{kg}$ per day GD14-20) as percent of vehicle control. (B) DBI/ACBP mRNA expression in prenatally treated and untreated males as percentage of females. TH thymus; SP spleen; AD adrenal gland; TE testis. THC, THD thymus of control and treated rats; SPC, SPD spleen of control and treated rats; ADC, ADD adrenal gland of control and treated rats. Asterisk: significant differences (for levels of significance, see Table 1).

complex tissue- and stage-specific pattern that strongly depends on the sex of the offspring. The rather distinct changes in DBI/ACBP mRNA contrast with the absence of detected changes in PBR mRNA expression. Both peptides are influenced by BDZ treatment in the adult organism (Mocchetti \& Santi 1991, Weizman et al. 1997), 
Table 2 Effect of prenatal diazepam exposure on PBR mRNA expression (mean \pm S.E.M. $)^{1}$

\begin{tabular}{|c|c|c|c|c|}
\hline & Males & & Females & \\
\hline & Prenatal vehicle & Prenatal diazepam & Prenatal vehicle & Prenatal diazepam \\
\hline GD20 ( $i$ & & & & \\
\hline Adrenal & $0.957 \pm 0.102$ & $1 \cdot 001 \pm 0 \cdot 128(4)$ & $0.988 \pm 0.076$ & $1 \cdot 026 \pm 0 \cdot 142(4)$ \\
\hline Testis & $0 \cdot 139 \pm 0 \cdot 021(4)$ & $0 \cdot 149 \pm 0 \cdot 019(4)$ & - & - \\
\hline PN14 (N & & & & \\
\hline Adult (N & & & & \\
\hline Thymus & $28 \cdot 9 \pm 5 \cdot 6(3)$ & $29 \cdot 2 \pm 4 \cdot 3(6)$ & $31 \cdot 1 \pm 5 \cdot 8(6)$ & $29 \cdot 8 \pm 7 \cdot 7(4)$ \\
\hline Spleen & $424 \cdot 0 \pm 134 \cdot 7(4)$ & $975 \cdot 1 \pm 292 \cdot 3$ & $305 \cdot 3 \pm 120 \cdot 9$ & $447 \cdot 4 \pm 168 \cdot 7(4)$ \\
\hline Adrenal & $224 \cdot 6 \pm 34 \cdot 4$ & $311 \cdot 1 \pm 34 \cdot 3(10)$ & $422 \cdot 3 \pm 59 \cdot 0(9)^{d}$ & $433 \cdot 9 \pm 85 \cdot 1(9)^{d}$ \\
\hline
\end{tabular}

${ }^{1} 4-10$ litters.

${ }^{2}$ In situ hybridization: (ROD); Northern blots: corrected optical density.

asex difference, controls $P=0 \cdot 022$, diazepam $P=0 \cdot 008$ (Bonferroni).

${ }^{b}$ Sex difference, controls and diazepam both $P<0 \cdot 001$ (Bonferroni).

${ }^{\mathrm{C}}$ Female diazepam different from control $P<0 \cdot 001$ (Bonferroni), general treatment effect $P<0 \cdot 001$.

${ }^{\mathrm{d}}$ Sex difference ANOVA $P=0 \cdot 007$.

but in the tissues studied DBI/ACBP regulation appears to be more sensitive during ontogeny.

Insofar as DBI/ACBP and PBR are thought to represent a ligand-receptor system at least in some tissues (Papadopoulos et al. 1997), one might hypothesize that immediate and delayed drug effects on the expression of the two proteins might be related to each other. Yet, an indication for a possible regulatory relationship between the two proteins was encountered only in the inverse relationship between DBI/ACBP mRNA and PBR mRNA in spleen (P14). Otherwise, the responses of DBI/ACBP mRNA and PBR mRNA expression to diazepam seemed largely independent of each other. The notion of an independent developmental regulation of the two proteins is also supported by different temporal and regional developmental patterns (Bürgi et al. 1999).

\section{Acknowledgements}

The investigation was supported by Swiss National Science Foundation grant no. 31-042366.94. We thank M E Lauber for helpful discussions on in situ hybridization, R Züllig and I Knüsel for their introduction to Northern blot analysis, and M Müller for skillful technical assistance. A preliminary report of this work was presented at the XXXIXth Annual Meeting of the International Society of Psychoneuroendocrinology, Trier, 2-6 August, 1998.

\section{References}

Amiri Z, Weizman R, Katz Y, Burstein O, Edoute Y, Lochner A \& Gavish M 1991 Testosterone and cyproterone acetate modulate peripheral but not central benzodiazepine receptors in rats. Brain Research 553 155-158.
Bell FP 1985 Inhibition of acyl-CoA:cholesterol acyltransferase and steroidogenesis in rat liver by diazepam in vitro. Lipids 20 75-79.

Benton L, Shan LX \& Hardy MP 1995 Differentiation of adult Leydig cells. Journal of Steroid Biochemistry and Molecular Biology 53 61-68.

Bürgi B, Lichtensteiger W, Lauber ME \& Schlumpf M 1999 Ontogeny of diazepam binding inhibitor/acyl-CoA binding protein mRNA and peripheral benzodiazepine receptor mRNA expression in the rat. Journal of Neuroendocrinology 11 85-100.

Bütikofer EE, Lichtensteiger W \& Schlumpf M 1993 Prenatal exposure of diazepam causes sex-dependent changes of the sympathetic control of rat spleen. Neurotoxicology and Teratology 15 377-382.

Elholm M, Bjerking G, Knudsen J, Kristiansen K \& Mandrup S 1996 Regulatory elements in the promoter region of the rat gene encoding the acyl-CoA-binding protein. Gene 173 233-238.

Fyrst H, Knudsen J, Schott MA, Lubin BH \& Kuypers FA 1995 Detection of acyl-CoA-binding protein in human red blood cells and investigation of its role in membrane phospholipid renewal. Biochemical Journal 306 793-799.

Garnier M, Boujrad N, Ogwuegbu SO, Hudsen JR Jr \& Papadopoulos V 1994 The polypeptide diazepam binding inhibitor and a higher affinity mitochondrial peripheral-type receptor sustain constitutive steroidogenesis in the R2C Leydig tumor cell line. Journal of Biological Chemistry 269 22105-22112.

Gavish M 1995 Hormonal regulation of peripheral-type benzodiazepine receptors. Journal of Steroid Biochemistry and Molecular Biology 53 57-59.

Guidotti A, Forchetti CM, Corda MG, Konkel D, Bennett CD \& Costa E 1983 Isolation, characterization, and purification to homogeneity of an endogenous polypeptide with agonistic action on benzodiazepine receptors. PNAS $\mathbf{8 0}$ 3531-3535.

Jefcoate CR, McNamara BC, Artemenko I \& Yamazaki T 1992 Regulation of cholesterol movement P450 scc in steroid hormone synthesis. Journal of Steroid Biochemistry and Molecular Biology 43 751-767.

Kellogg CK 1988 Benzodiazepines: influence on the developing brain. Brain Research 73 207-228.

Kerkoff C, Beuck M, Threige-Rasmussen J, Spener F, Knudsen J \& Schmitz G 1997 Acyl-CoA binding protein (ACBP) regulates acyl-CoA:cholesterol acyltransferase (ACAT) in human mononuclear phagocytes. Biochimica et Biophysica Acta 1346 163-172. 
Knudsen J, Mandrup S, Rasmussen JT, Andresen PH, Poulsen F \& Kristiansen K 1993 The function of acyl-CoA binding protein (ACBP) regulates acyl-CoA:cholesterol acyltransferase (ACAT) in human mononuclear phagocytes. Molecular and Cellular Biochemistry 123 129-138.

Mandrup S, Hummel R, Raven S, Jensen G, Andreasen HP, Gregersen N, Knudsen J \& Kristiansen K 1992 Acyl-CoA-binding protein/diazepam-binding inhibitor gene and pseudogenes. A typical housekeeping gene family. Journal of Molecular Biology 228 1011-1022.

Mocchetti I \& Santi MR 1991 Diazepam binding inhibitor peptide: cloning and gene expression. Neuropharmacology 30 1365-1371.

Mogensen IB, Schulenberg H, Hansen HO, Spener F \& Knudsen J 1987 A novel acyl-CoA-binding protein from bovine liver. Biochemical Journal 241 189-192.

Papadopoulos V, Amri H, Boujrad N, Cascio C, Culty M, Garnier M, Harwick M, Li H, Vidic B, Brown AS, Reversa JL, Bernassau JM \& Drieu K 1997 Peripheral benzodiazepine receptor in cholesterol transport and steroidogenesis. Steroids 62 21-28.

Rasmussen JT, Rosendal J \& Knudsen J 1993 Interaction of acyl-CoA binding protein (ACBP) on processes for which acyl-CoA is a substrate, product or inhibitor. Biochemical Journal 293 907-913.

Rasmussen JT, Faergeman NJ \& Knudsen J 1994 Acyl-CoA-binding protein (ACBP) can mediate intermembrane acyl-CoA transport and donate acyl-CoA for beta oxidation and glycerolipid synthesis. Biochemical Journal 299 165-170.

Sambrook J, Fritsch EF \& Maniatis T 1989 Molecular Cloning, edn 2, vol 1. Cold Spring Harbor, New York: Cold Spring Harbor Laboratory Press.

Schlumpf M, Richards G, Lichtensteiger W \& Möhler H 1983 An autoradiographic study of the prenatal development of benzodiazepine-binding sites in rat brain. Journal of Neurosciences 3 $1478-1487$.

Schlumpf M, Parmar R, Ramseier HR \& Lichtensteiger W 1990 Prenatal diazepam induced immunosuppression: possible involvement of peripheral benzodiazepine site. Developmental Pharmacology and Therapeutics 15 178-185.

Schlumpf M, Lichtensteiger W \& Ramseier HR 1993a Diazepam treatment of pregnant rats differentially affects interleukin- 1 and interleukin-2 secretion in their offspring during different phases of postnatal development. Pharmacology and Toxicology 73 335-340.

Schlumpf M, Parmar R \& Lichtensteiger W 1993b Prenatal diazepam induced persisting downregulation of peripheral $(\omega 3)$ benzodiazepine receptors on rat splenic macrophages. Life Sciences $\mathbf{5 2}$ 927-937.

Schlumpf M, Parmar HR, Bütikofer EE, Inderbitzin S, Salili AR, Schreiber AA, Ramseier H, Van Loveren H \& Lichtensteiger W 1995 Delayed developmental neuro- and immunotoxicity of benzodiazepines. Archives of Toxicology 17 (Suppl) 261-287.

Swinnen JV, Esquenet M, Heyns W, Rombauts W \& Verhoeven G 1994 Androgen regulation of the messenger RNA encoding diazepam-binding inhibitor/acyl-CoA-binding protein in the human prostatic adenocarcinoma cell line LNCaP. Molecular and Cellular Endocrinology 104 153-162.

Swinnen JV, Vercaeren I, Esquenet M, Heyns W \& Verhoeven G 1996 Androgen regulation of the messenger RNA encoding diazepam-binding inhibitor/acyl-CoA-binding protein in the rat. Molecular and Cellular Endocrinology 118 65-70.

Swinnen JV, Alen P, Heyns W \& Verhoeven G 1998 Identification of diazepam-binding inhibitor/acyl-CoA-binding protein as a sterol regulatory element-binding protein-responsive gene. Journal of Biological Chemistry 273 19938-19944.

Todaro GJ, Rose TM \& Shojab M 1991 Human DBI (endozepine): relationship to a homologous membrane associated protein (MA-DBI). Neuropharmacology 30 1373-1380.

Weizman R, Dagan E, Snyder SH \& Gavish M 1997 Impact of pregnancy and lactation on $\mathrm{GABA}_{\mathrm{A}}$ receptor and central-type and peripheral-type benzodiazepine receptors. Brain Research $\mathbf{7 5 2}$ 307-314.

Woods MJ, Zisterer DM \& Williams DC 1996 Two cellular and subcellular locations for the peripheral-type benzodiazepine receptor in rat liver. Biochemical Pharmacology 51 1283-1292.

Received 7 September 1999

Revised manuscript received 9 February 2000

Accepted 24 February 2000 